\title{
Development of a next-generation temporary fixing agent for electronic materials
}

\author{
Hiroyuki Kurimura, Jun Watanabe, Kazuhiro Oshima, Kimihiko Yoda and Norihiro Shimizu
}

We have developed a next-generation temporary fixing agent for electronic materials. This fixing agent allows for film peel-off via immersion in hot water while retaining strong adhesiveness, which can resist various forms of processing. Although thermoplastic adhesives (known as waxes) have been previously used as temporary fixing agents, problems related to their safety and workability were observed because they melt at high temperatures during the adhesion process. In addition, environmental issues were encountered due to the use of organic solvents in the cleaning process that was performed after peel-off. Conversely, our temporary fixing agent is expected to compensate for such limitations because it possesses advantageous characteristics, such as curing capabilities over short durations at room temperature using redox polymerization or photopolymerization, peel-off via immersion in hot water, and a film-type peel-off. Such fixing agents are becoming the de facto standard for use in the processing of capacitive touch sensors in glass covers, for example, in smartphones and tablet PCs. Polymer Journal (2016) 48, 565-570; doi:10.1038/pj.2015.83; published online 6 January 2016

\section{INTRODUCTION}

Temporary fixing agents are used for temporarily fixing workpiece materials on processing tools with high accuracy during ceramic and optical element processing. Thermoplastic wax is an example of a typical temporary fixing agent; widely used standard waxes include pine tar and waxes from the paraffin series. ${ }^{1}$ Because high temperatures are required for adhesion and peel-off, the involved heating and cooling processes not only require time but are also damaging. There are additional issues such as the requirement for immersion in organic solvents to dissolve the wax after processing, which causes potential environmental concerns and requires a significant amount of work to perform the cleaning process. ${ }^{2,3}$

In this study, we developed a next-generation temporary fixing agent for electronic materials. This is a completely new concept, and the temporary fixing agent acts as a substitute for wax; the product name is TEMPLOC. The characteristics of this fixing agent are as follows: it has the ability to cure over a short duration at room temperature using photopolymerization or redox polymerization; it does not involve the use of organic solvents because it can be easily peeled off using hot water; and it has a film-type peel-off, so it is capable of protecting work without leaving any deposits. Such characteristics help reduce environmental concerns and damage to components because curing can occur over a short duration without the application of heat. ${ }^{4-8}$ Therefore, we propose that this temporary fixing agent will help reduce costs and simplify the cleaning process. Figure 1 uses an example related to dicing with optical glass to show a comparison of an electronic material manufacturing process using either the next-generation temporary fixing agent or a standard wax.
In addition, when this next-generation temporary fixing agent is peeled off and used as a protective film, a new lamination-adhesion production process is developed to grind and cut electronic components, such as glass sheets. The use of several lamination-adhesive (fixed) layers should improve yield and productivity. Here, we present the details of this study.

\section{EXPERIMENTAL PROCEDURE}

Creating a next-generation temporary fixing agent that can strongly adhere (fixed) and be easily peeled off as a film

Denki Kagaku Kogyo Kabushiki Kaishya manufactures and sells structural adhesives that are classified as second-generation acrylic adhesives. ${ }^{1,4-8}$ After more than 30 years of development aimed at achieving reliability and high performance, the fundamental technology for strong adhesion (fixation) has been used to develop various material design techniques that can withstand high temperatures and high humidity and help satisfy the demand for high-level electronic products. ${ }^{9}$ During this development, we considered the possibility of creating adhesives (temporary fixing agents) that would strongly adhere but easily peel off at high temperature and high humidity. This approach was the complete opposite of the previous material design concepts that provided resistance against high temperatures and high humidity. Thus, we arrived at the idea of rapidly peeling an adhesive off as a film using hot water as a trigger.

This process was achieved by allowing hot water to penetrate from the outside into the adhesion interface, thereby allowing for control of thermal expansion and the internal stresses that form, while curing shrinkage by implementing an appropriate cross-linked structure. 
a

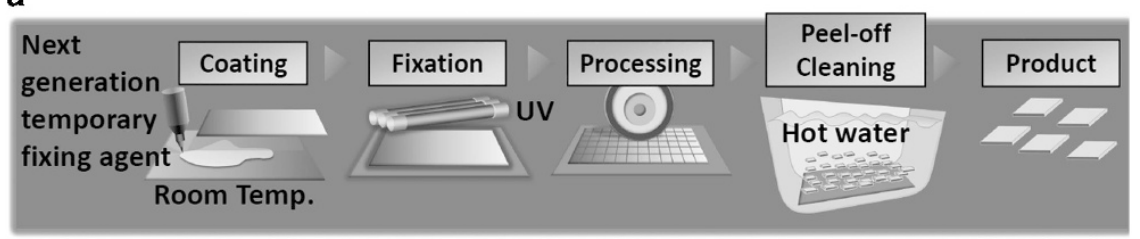

b

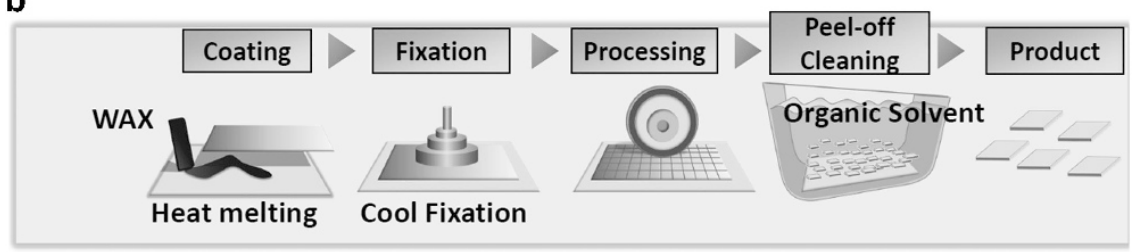

Figure 1 An electronic material manufacturing process: a comparison between the (a) next-generation temporary fixing agent and (b) conventional wax. In a process using conventional wax (b), high temperature is required during fixing, and an organic solvent is used for peel off and cleaning. In contrast, the next-generation temporary fixing agent (a) can be cured and fixed at room temperature over a short period of time by using photopolymerization or redox polymerization and can be peeled off as a film by immersion in hot water.

UV

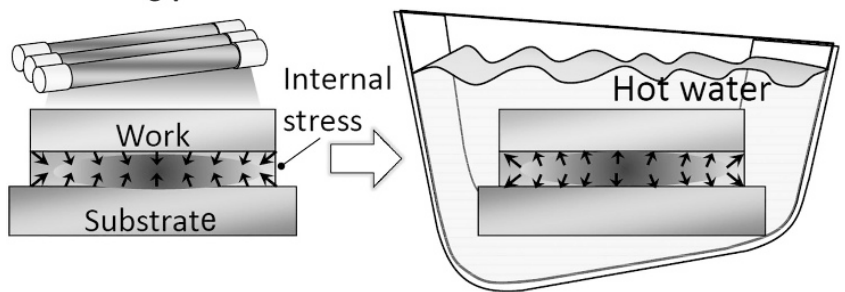

Figure 2 Peel-off mechanism for the next-generation temporary fixing agent. When a component and substrate are fixed with a next-generation temporary fixing agent, internal stress is generated in the fixing agent (a). By immersing the adhesion product in hot water, the generated internal stress is released, the cured body of the next-generation temporary fixing agent undergoes volume expansion, and water penetrates into the adhesion interface, whereby the adhesively fixed body can be peeled off (b).

Evaluation of this concept showed that an adhesive (temporary fixing agent) could achieve a balance between easy peel-off as a film and strong adhesion (fixation). Figure 2 shows the peel-off mechanism of the next-generation temporary fixing agent. By laminating an optical element (work) to a support using the temporary fixing agent, the work was temporarily adhered (fixed) either by combining two liquids (redox polymerization) or by applying UV exposure (UV polymerization).

Our next-generation temporary fixing agent incurs internal stress because of curing shrinkage. Under such conditions, it is impossible to peel off the agent even if cutting or grinding is performed. However, if immersed in hot water at $80^{\circ} \mathrm{C}$, the next-generation temporary fixing agent undergoes volume expansion, causing internal stress and distortion to be released and allowing film-type peel-off. Next, we explain the various techniques that are the basis for this process.

Design of a film-type, hot water and short-time peel-off fixing agent that uses the interior distortion and stress that accompanies curing shrinkage

To increase the resistance of adhesives to high temperatures and high humidity, material design typically aims to reduce the interior distortion and stress caused by the volume shrinkage that occurs during curing. ${ }^{10}$ However, we moderately increased the internal distortion and stress during the curing shrinkage process by regulating

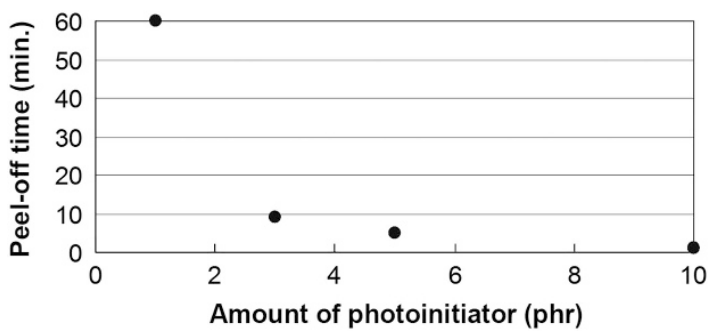

Figure 3 Relationship between peel-off time and amount of photoinitiator in the next-generation temporary fixing agent.

the cross-linked structure (and the density therein) that was formed during curing. The internal stress and distortion were subsequently reduced when the adhesion product was immersed in hot water. Thus, water penetrates into the adhesion interface, allowing the adhesion product to be peeled off. Accordingly, we have developed a peel-off effect that does not sacrifice adhesivity. In general, we can consider that internal stress increases when the amount of shrinkage in the cured material increases, that is, as the amount of photoinitiator increases with respect to photopolymerization. Next, we conducted a detailed investigation of the peel-off behavior by hot water immersion of adhesion products with varying amounts of photoinitiators in their light-curing acrylic adhesives. For this step, we applied $0.1 \mathrm{~g}$ of the next-generation temporary fixing agent, consisting of several acrylic monomers and photoinitiators, to heat-resistant Pyrex (trademarked product) glass $(25 \mathrm{~mm} \times 25 \mathrm{~mm} \times 2.0 \mathrm{~mm})$, and adhered it to a sodalime glass $(150 \mathrm{~mm} \times 150 \mathrm{~mm} \times 1.7 \mathrm{~mm})$ support. We then used a UV irradiation device with a metal-halide lamp to perform the adhesion step. A UV cumulative illuminator (UV meter UVPF-Al, manufactured by EYE GRAPHICS Co., LTD, Sumida-ku, Tokyo, Japan), which uses a 365-nm optical receiver, was employed to set the level of

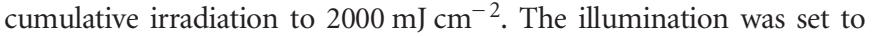
$160 \mathrm{~mW} \mathrm{~cm}^{-2}$. We then immersed the obtained test product in hot water $\left(80^{\circ} \mathrm{C}\right)$ and measured the time required for the heat-proof Pyrex glass to peel off of the blue-board glass. As shown in Figure 3, we observed that when placed in hot water, the peel-off time decreased as the amount of photoinitiator increased. Furthermore, we developed a technique in which the temporary fixing agent is not dissolved in water during peel-offs and can be used as a film. This was achieved by 
a

Main Component: hydrophilicity

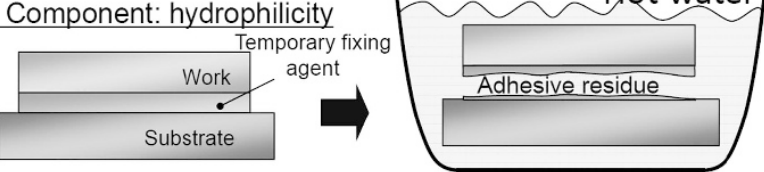

b

Main Component: hydrophobicity
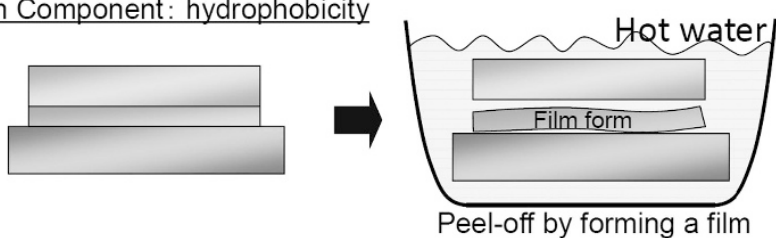

Figure 4 Concept of the material design for peel-off film. When using a hydrophilic main component, as shown in (a), the temporary fixing agent was not sufficiently dissolved when immersed in hot water to allow for peel off, and an adhesive residue was formed. When using a hydrophobic component, as shown in (b), the temporary fixing agent formed a film in hot water and peeled off without leaving an adhesive residue.

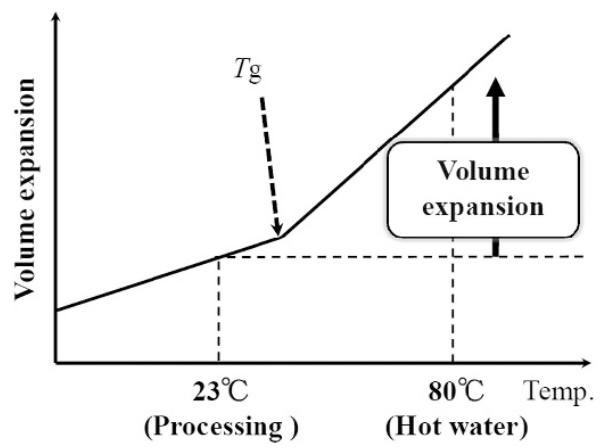

Figure 5 Improving peelability through regulation of the glass-transition temperature. The glass-transition temperature of the next-generation temporary fixing agent was regulated to increase the processing temperature of the temporary fixing agent. The glass-transition temperature was reduced, and by increasing the peel-off temperature after immersion of the temporary fixing agent in hot water, the volume of expansion was increased to enhance peel off.

controlling the density and cross-linked structure by optimizing the molecular structure, with respect to the polarity and number of functional groups, in the cross-linked monomer (Figure 4).

Design of a short-time peel-off in hot water using thermal expansion characteristics

We attempted to regulate the thermal expansion characteristics of the cured temporary fixing agent layer during adhesion (fixation). ${ }^{10} \mathrm{We}$ hypothesized that if we could increase the volume of expansion in the cured temporary fixing agent layer by increasing its temperature after submersion in hot water, then we could expect that peel-off would be improved (Figure 5). In fact, by increasing the amount of thermal expansion using a design with a lowered glass-transition temperature in the temporary fixing agent layer, we were able to easily introduce a gap between the work and the cured temporary fixing agent layer, which allowed us to improve peelability upon immersion of the interface in hot water (Figure 6).

\section{EXAMPLES FOR APPLYING NEXT-GENERATION FIXING AGENTS}

The application of this photocuring, next-generation fixing agent significantly improves the polishing and dicing processes used

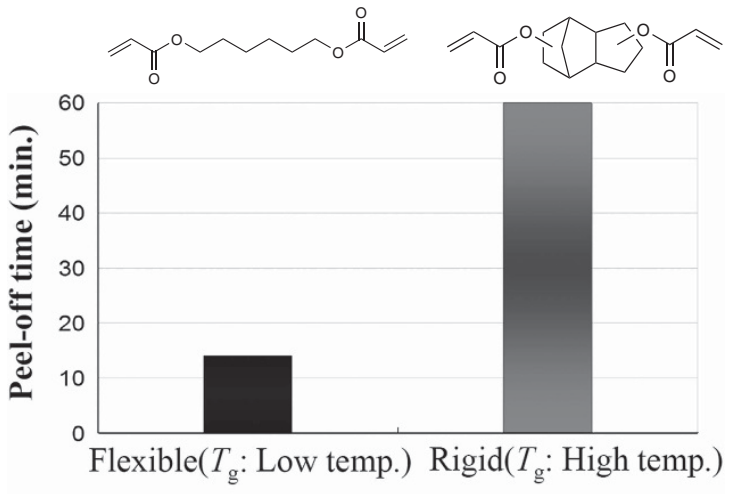

Cross-linking monomer species

Figure 6 Relationship between cross-linking monomer species and peel-off time.

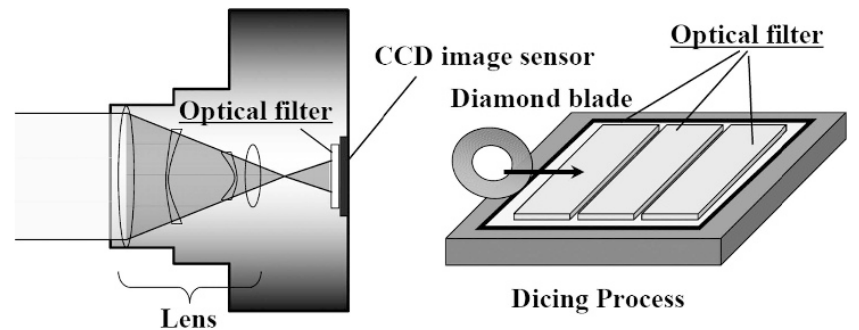

Figure 7 Optical filter dicing process for use in digital cameras.

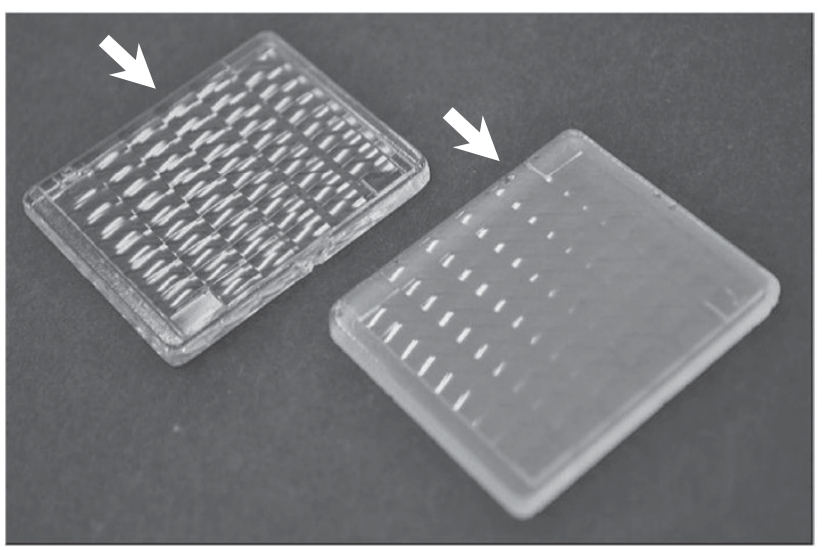

Figure 8 Fly-eye lens for use in projectors. The site marked with an arrow is the surface coated with the next-generation temporary fixing agent.

on lenses, prisms, mirrors and filters (which are used in the optoelectronics field for various displays and digital cameras). ${ }^{4-8}$ Next, we outline some typical application examples.

Application example 1: digital camera optical low-pass filter The temporary fixing agent was applied to the dicing process for optical low-pass filters of a digital camera ${ }^{1}$ (Figure 7 ). When compared with processes that use wax, this product reduced the peel-off time from $6 \mathrm{~h}$ to $7 \mathrm{~min}$ and reduced the residual material that remained on the work. This reduced the total number of processing steps required for cleaning from seven to three and the total processing time from 10 to $2 \mathrm{~h}$. 
Application example 2: projector fly-eye lens

The temporary fixing agent was applied to the polishing and grinding processes used on light-collecting fly-eye lenses installed in projectors. ${ }^{1}$ Although we observed work with some unevenness on one side of the fly-eye lens, as shown in Figure 8, the fly-eye lens was easily adhered to the glass support with high accuracy. The fixing agent could then be used to perform UV irradiation on the uneven side. Peel-off, without residue, was easy when the back of the uneven side was immersed in hot water for $10 \mathrm{~min}$ after grinding and polishing.

\section{Application example 3: silicon wafers for use in solar-powered batteries}

The application of the next-generation temporary fixing agent is not limited to the processing of transparent materials, such as glass.

a

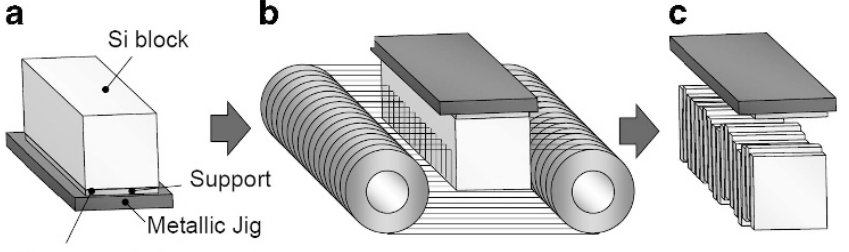

Temporary fixing agent

Figure 9 Silicon block slicing process with a multi-wire saw. (a) Fixing the silicon block and support with the temporary fixing agent. (b) Slicing the silicon block with a multi-wire saw. (c) Separating silicon wafers.

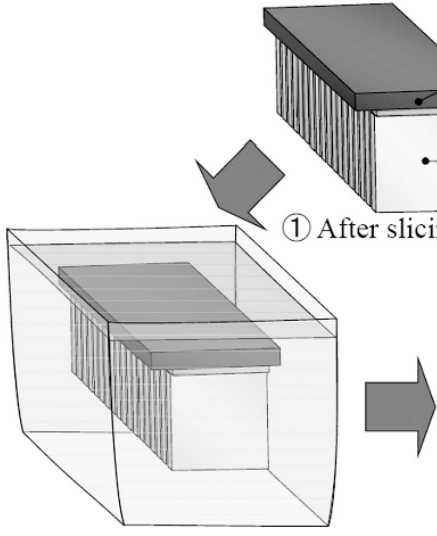

(2) Immersed in hot water

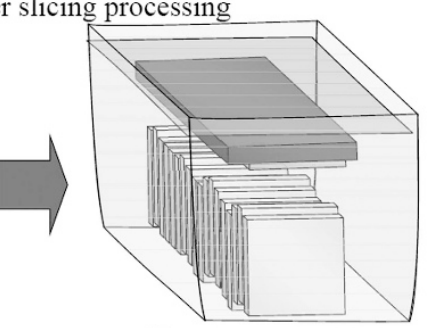

(3) Silicon wafer peeled off
Figure 10 Silicon wafer processed by peel-off of the next-generation temporary fixing agent.
Temporary fixing agents (for example, SOLARLOC) that utilize redox curing have also been applied to the processing of opaque materials. As illustrated in Figure 9, silicon wafers applied in solar cells were cut from a rectangular parallelepiped-shaped silicon block with a slicing device called a multi-wire saw. ${ }^{11,12}$ This method enabled the simultaneous production of several identically shaped silicon wafers through a simple process. In accordance with increasing the production of solar-powered batteries, important attention has recently been focused on improving the processing accuracy and production efficiency. Needs have arisen with regard to resolving issues associated with using mainstream epoxy-based adhesives as temporary fixing agents; for example, there is a need to reduce the curing reaction time required to fix silicon blocks and ingots on supports and to eliminate environmental problems arising from the liquids used for peel-off.

For the processing of silicon blocks and ingots for use with solarpowered batteries, the application of main agents comprising two liquids in temporary fixing agents, which use redox polymers in their curing systems, continues to progress. For example, silicon blocks undergo a slicing process using wire saws where these temporary fixing agents are used in a series of processes until the silicon wafers are peeled off. Compared with epoxy-based adhesives, temporary fixed adhesion between a support and a silicon block (Figure 10) indicates that such temporary fixing agents have a significantly shorter curing duration and stronger adhesive force. Moreover, because of their much smaller viscosity compared with the epoxy adhesives, the temporary fixing agents have a good workability and are easy to manage, similar to the two-liquid main agent-type acrylic adhesives,

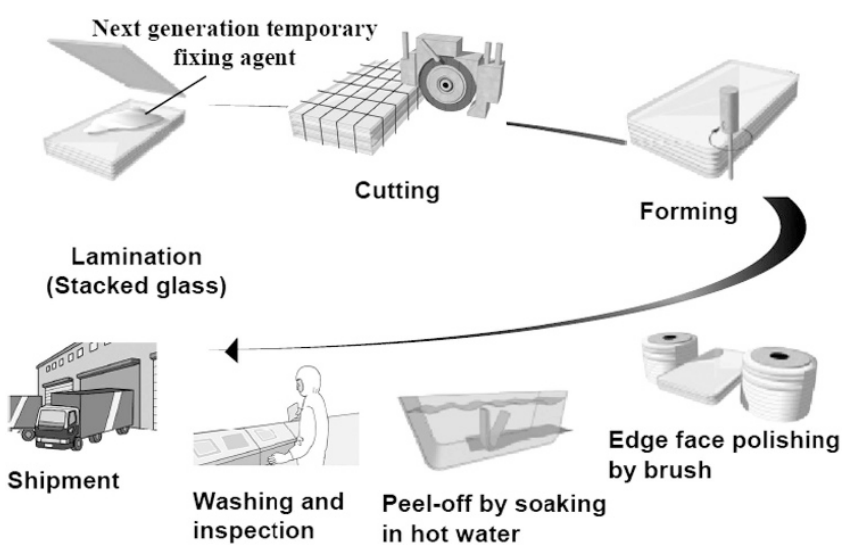

Figure 12 OGS lamination-adhesion process using the next-generation temporary fixing agent.

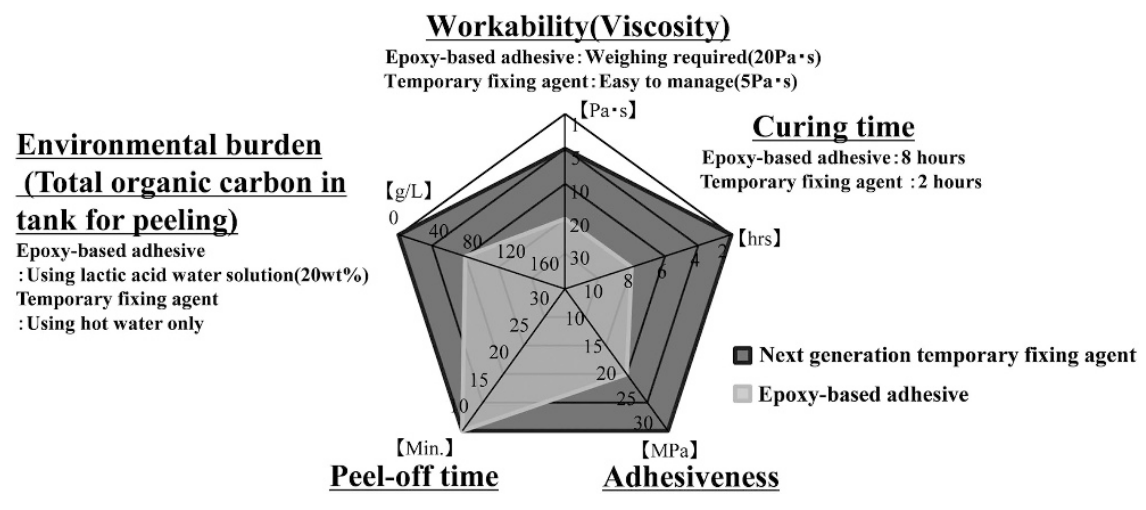

Figure 11 Comparison of the characteristics of redox curing, next-generation temporary fixing agents and epoxy-based adhesives. 
which contributed to improvements in workability and productivity during silicon wafer processing. In addition, these temporary fixing agents differ from epoxy-based adhesives because they do not use lactic acid and only require hot water to peel off; thus, they can mitigate environmental concerns. For example, the total organic carbon content in the tank used for peeling is much higher for epoxy-based adhesives than for these temporary fixing agents (Figure 11). ${ }^{13}$

Application example 4: creating a new lamination-adhesive process Currently, because the touch panels used in elongated smart phones are continually becoming thinner, providing high-definition displays, ${ }^{1,14-17}$ One-Glass-Solution (OGS) touch panels equipped with reinforced glass with integrated sensors that incorporate touch sensor capabilities are expected to become mainstream in the future.

The OGS production process is used, via a processing step, to perform separation after printing delicate ITO films, insulation films and $\mathrm{Al}$ wires on a reinforced large glass side. However, it is difficult to practically apply this separation using traditional processing techniques because of the need to process reinforced glass one sheet at a time, which results in several problems, such as poor processing accuracy and low productivity. Therefore, we have developed a new process for dicing and polishing, which includes laminating and applying adhesion (fixation) to several sheets of electronic components, such as glass, using this temporary fixing agent.

This process is advantageous because of the film-like peeling, which is a characteristic feature of this temporary fixing agent and represents an original processing technique with the benefit of 'no mutual contact or damaging of glass components' during peel off. In addition, this new lamination-adhesion processing technique was the first in the world to be implemented for mass production of an OGS dicing and grinding process in 2010. Since then, its use has spread globally, pioneering the creation of a new market that is expected to experience significant growth in the future.

Figure 12 shows an OGS lamination-adhesion process that uses this next-generation temporary fixing agent. This processing technique was able to stabilize strength and was capable of performing an all-inclusive cutting process by laminating and applying adhesion to several sheets of glass substrate. In addition, because this temporary fixing agent is capable of protecting a delicate touch sensor during processing, it is also capable of increasing production yield and lowering production costs.

Because this lamination technique has spread domestically, over 10000 sheets of OGS have been shipped from the production facility. Moreover, the process has continued to spread overseas (Korea, China and Taiwan), becoming a world leader and the default method for OGS processing.

\section{CONCLUSION}

Next-generation temporary fixing agent adhesives, which are capable of retaining strong adhesiveness, resist various types of processing and result in film-type peel-off by immersion in hot water, have been developed and applied in various electronic processes. In addition, new lamination-adhesion processing methods that use nextgeneration temporary fixing adhesives, for example, the processing of touch panels used in smart phones, are gradually becoming the default standard. However, there are demands for adhesives that can provide both hot water peel-off abilities and heat resistance at temperatures above $230^{\circ} \mathrm{C}$. Such adhesives are used in the production of semiconductors and touch panel films. Addressing this demand is a major challenge for which we continue to conduct intensive research. In addition, we plan to further advance the design of materials that meet current demands and needs, for example, touch panel processing and the previously mentioned electronic processes.

\section{CONFLICT OF INTEREST}

The authors declare no conflict of interest.

\section{ACKNOWLEDGEMENTS}

We received 'The Award of the Society of Polymer Science, Japan (Technology) 2012' awarded by the Society of Polymer Science, Japan, for the study of the 'development of next generation temporary fixing agents for use with electronic processes. ${ }^{1}$ For receiving this award, we would like to express our sincere gratitude to all those who collaborated and provided support.

1 Shimizu, N., Watanabe, J., Oshima, K., Yoda, K. \& Kurimura, H. Development of next generation temporary immobilizing agent for electronics process. Polymer Preprints 62 ROMBUN N0.2A09IL (2013) (in Japanese).

2 Haraga, K. Application of hot melt adhesives 3.6. Electric and electronic devices and hot melt. Adhes. Technol. 14, 69-73 (1994) (in Japanese).

3 Nomura, M. Technology for visible light hardening water-soluble temporary fixing agent. JET/ 48, 147-149 (2000) (in Japanese).

4 Ogata, Y. \& Oshima, K. Ecologically friendly adhesive "TEMPLOC" for temporary fixing. JETI 54, 69-71 (2006) (in Japanese).

5 Ogata, Y. The Group for eco-friendly adhesive products of our Company-an environmentally adaptable adhesive "HARDLOC" and its development. Kougyou Tosou 209, 39-44 (2007) (in Japanese).

6 Ogata, Y. Rediscovery of materials-Series, Part IV Novel soft materials 5. New types of temporary fixing adhesives. Adhes. Technol. 26, 22-25 (2007) (in Japanese).

7 Oshima, K. Temporary adhesive for processing of electronic parts. Fine Chemicals 37 11-16 (2008) (in Japanese).

8 Denki Kagaku Kogyo Kabushiki Kaisha. High performance temporary fixing adhesive "TEMPLOC". Poly File 48, 33-35 (2011) (in Japanese).

9 Ogata, Y. Features and usage of construction adhesive "HARDLOC". JETI 53 , 133-135 (2005) (in Japanese).

10 Kurimura, H., Kanai, T. \& Oshima, K. Adhesive composition and method for temporary fixing member by using the same. PCT Pub. No. WO2008/018252 (2008).

11 Sakata, N. "Easy-to-Understand solar power generation" for beginners, solar cell manufacturing technology and evaluation apparatus, solar cell wafer manufacturing lines. Denshi Zairyou 47, 76-80 (2008) (in Japanese).

12 Suwabe, H. Cut processing technology of solar cell wafers. Sci. Machine 64, 816-822 (2012) (in Japanese)

13 Hisha, Y. \& Oshima, K. Temporary fixing agent. Japanese Patent 2010-248395. (2010) (in Japanese).

14 Saeki, S. Emergence of new touch panels. Redesign of an industrial landscape. Nikkei Electronics 1082, 65-73 (2012) (in Japanese).

15 Oshima, K. Technical trends of "Reworkable-reparable" materials, glass processing adhesives for touch panels. Material Stage 12, 37-39 (2012) (in Japanese).

16 Tomioka, T. Adhesives peelable when requested. Nikkei Monozukuri 684 68-77 (2011) (in Japanese).

17 Oshima, K. A novel glass processing by using "TEMPLOC" in touch panel. New Glass 27, 17-22 (2012) (in Japanese). 


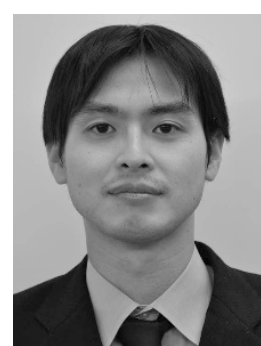

Hiroyui Kurimura was born in Tokyo Prefecture, Japan, in 1977. He received his Master degree from Graduate School of Science and Engineering, Waseda University in 2002. In the same year he joined Denka Co. Ltd (Japan) and started his R\&D at Central Research Institute. He was engaged in the R\&D of styrene-butadiene copolymer (2002-2004). Since 2004, he has been mainly engaged in the R\&D of acrylic materials for electronic materials, such as thermal interface materials (2004-2005) and acrylic-based adhesives including temporary fixing agent (2005- ). He was a recipient of The Award of the Society of Polymer Science, Japan (2013).

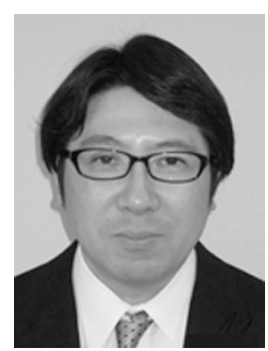

Jun Watanabe was born in Kanagawa Prefecture, Japan, in 1965. He received his Master degree from Faculty of Engineering, The University of Tokyo in 1990. In the same year he joined Denka Co. Ltd (Japan) and started his R\&D at Central Research Institute. He was engaged in the R\&D of styrene-based polymers (1990-2004), such as fire retardant styrene-based copolymer, rubber-reinforced styrene-based copolymer and styrene-butadiene block copolymer. From 2004, he was mainly engaged in the R\&D of acrylic-based adhesives including temporary fixing agent (2004-2011). After establishing the technology of the temporary fixing agent, he is currently a general manager at the Advanced Technologies Research Institute. His current research interests include functional polymeric materials. He was a recipient of Technology Award of the Adhesion Society of Japan in 2011 and The Award of the Society of Polymer Science, Japan (2013).

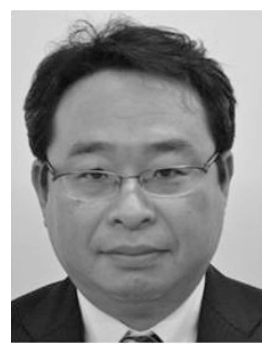

Kazuhiro Oshima was born in Mie Prefecture, Japan, in 1969. He received his Master degree from Graduate School of Engineering, Kagoshima University in 1994. In the same year he joined Denka Co. Ltd (Japan) and started his R\&D at Advanced Research Department. He was engaged in the R\&D of Hyaluronic acid, Polystyrene (1994-2000). Since 2000, he has been mainly engaged in the R\&D of Adhesives known as HARDLOC and thermal interface materials. He was a recipient of The Award of the Society of Polymer Science, Japan (2013).

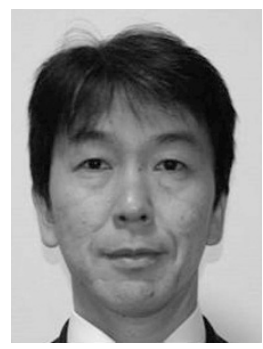

Kimihiko Yoda was born in Nagano Prefecture, Japan, in 1969. He graduated from West Virginia University in 1992. He joined Denka Co. Ltd (Japan) in 1992. He was engaged in the R\&D of Adhesives known as HARDLOC until 2012. After that he has been engaged in the production section of dicing tapes used for semiconductor machining. From 2014, he had been engaged in the R\&D of metal base circuit boards. He was a recipient of Technology Award of the Adhesion Society of Japan in 2011 and The Award of the Society of Polymer Science, Japan (2013).

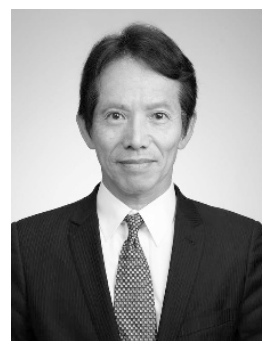

Norihiro Shimizu was born in Japan in 1955. He received his Master degree from the graduate school and faculty of engineering, Chiba University in 1980. In the same year he joined Denka Co. Ltd (Japan) and started his career in charge of Polychloroprene research in the R\&D department, Ohmi plant. He studied under the guidance of Professor A.N. Gent at the Institute of Polymer Science at the University of Akron, OH in USA from 1983 to 1985. After returning to Japan, he was engaged in studying the structural analysis programs in Kozo Keikaku Engineering Inc. He also developed pressure resistance PET bottles with self-standing design for carbonated drinks, new transparent styrene graft polymers and block copolymers. He was transferred to the Electronic Materials Institute as the Director in 2004 and he developed new thermal conductive materials and process, adhesives, temporary adhesives, metal circuit boards, etc. Since 2013, he has been assigned as managing executive director in charge of R\&D, new business development and IP. He is a recipient of The Award of the Society of Polymer Science, Japan (2013). He is the author and coauthor of a few original papers, reviews, books and many patents. 